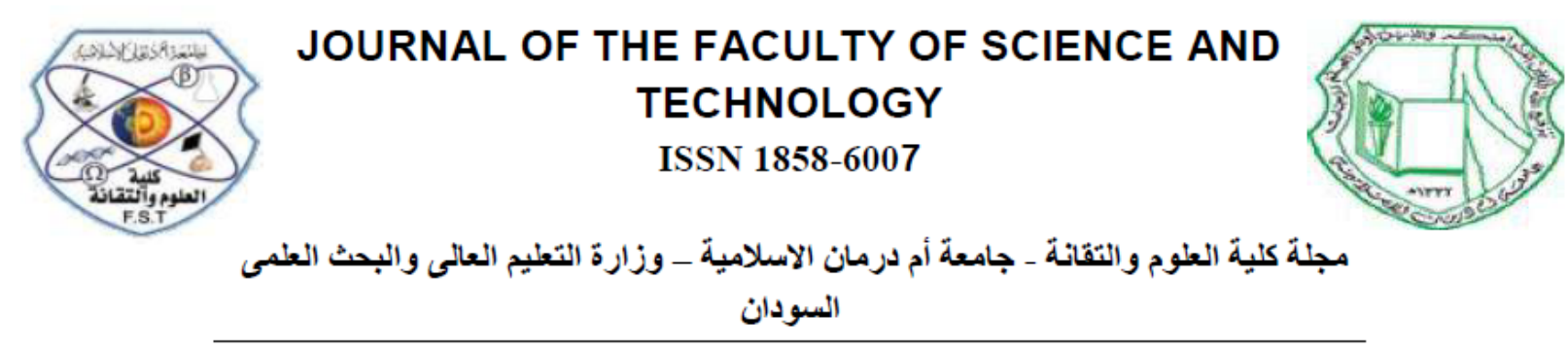

Research article

\title{
Band Structure of Sr Doped into Bulk Wurtzite ZnO: A DFT Study
}

\author{
Abdalla Abdelrahman Mohamed $^{1}$, Tasneem Babiker Abdalrahman ${ }^{2}$ \\ ${ }^{1}$ E-mail: abdallam194@gmail.com \\ ${ }^{2}$ E-mail: tasneembab@gmail.com
}

\begin{abstract}
This work investigates the structural and electronic properties of pure and $\mathrm{Sr}$-doped $\mathrm{ZnO}$ using first principles density functional calculations (DFT). The calculations were carried out using GGA-BLYP functional. This functional underestimates the band gap value in semiconductors but does not affect the accuracy of the related properties of the crystals. The Sr-doping caused increase in lattice volume and slight distortions at the unit cell parameters in a wurtzite structure. The doping process presented increase in the band-gap energy $\mathrm{E}_{\mathrm{g}}$ at low percentages $25 \%, 37.5 \%$ and 50\% with indirect bang gap and direct band gap at high percentages $62.5 \%, 75 \%, 87.5 \%$ and $100 \%$. which we can called it wide indirect band gap. These results can be use as a foundation for more in depth calculations which can be used on optical and Photo-catalytic applications.
\end{abstract}

Keywords: Doping; DFT; Wurtizte; Wide Semiconductor.

\section{Introduction}

$\mathrm{ZnO}$ can be considered as an 'old' semiconductor which has been compelling research attention for a long time because of its applications in many scientific and industrials areas. $\mathrm{ZnO}$ is a wide direct band gap semiconductor with $\mathrm{Eg} \approx 3.436 \mathrm{eV}$ at $\mathrm{T}$ $=0 \mathrm{~K}$ and $(3.37 \pm 0: 01) \mathrm{eV}$ at room temperature (Klingshirn et al. 2010). Wurtizte $\mathrm{ZnO}$ crystal structure is the most stable state with lattice parameters a 3.249

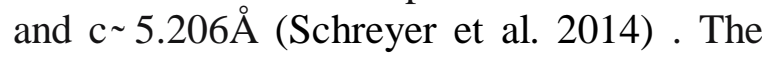
$\mathrm{Zn}$ atoms are tetrahedrally coordinated with four $\mathrm{O}$ atoms, where the $\mathrm{Zn}$ d-electrons hybridize with the $\mathrm{O}$ p-electrons.

Often, the first-principles calculation based on density functional theory (DFT) is used to evaluate the different properties of pure and doped $\mathrm{ZnO}$. Several works has employed DFT with Generalized Gradient Approximation PBE, LDA and other exchange-correlation functions (Mohamad et al. 2017), (Shen et al. 2008), (Kalay et al. 2009),(Mousavi 2017) and the references therein.

Not too much works has been investigates $\mathrm{Sr}$ doped $\mathrm{ZnO}$. Experimental investigation of structural and optical properties of nano thin films of $\mathrm{ZnO}$ and $\mathrm{Sr}$-doped $\mathrm{ZnO}$ onto glass substrates synthesized by a two-step chemical bath deposition technique revealed that The films are polycrystalline in nature with hexagonal phase having (002) 
preferential orientation and The shifts in optical band gap of the SZO films are 2.35 (Vijayan et al. 2008)

First-principles calculations have been performed to determine the effects of $\mathrm{Sr}$ doping on the structural, electronic, and optical properties of $\mathrm{ZnO}$ nanotubes (Moradian and Shahrokhi 2013); It is found that all doped systems are exothermic and stable, but by increasing Sr-doping concentration the formation energy increased The semiconducting energy gap of Sr-doped single walled $\mathrm{ZnO}$ nanotubes is bigger than their pristine $\mathrm{ZnO}$ nantube. This study also showed that the systems have negative formation energy for all impurity concentrations and that all systems have also direct band gap at $\Gamma$-point and are nonmagnetic. Mahmood et al employed the PBE exchange-correlation function parameters in GGA to study the electronic structure and optical properties of the pure and $\mathrm{Sr}$-doped $\mathrm{ZnO}$; they find out that $\mathrm{The} \mathrm{Sr}$ doping significantly affects the electronic band gap, The band gap was observed to increase with increasing $\mathrm{Sr}$ content(Mahmood et al. 2017).

In this paper, as the use of $\mathrm{ZnO}$ in a wide range of applications would not be possible without the doping process, which can be used to change drastically the material properties, we use density functional theory (DFT) calculations under GGA allied to BLYP exchange correlation functional to study the Sr-doping at $12.5 \%, 25 \%, 37.5 \%$, $62.5 \%, 75 \%$ and $87.5 \%$ concentration into bulk wurtizte zinc oxide which expected to change the band structure properties in particular direct and indirect band gap of the material.

\section{Methods}

We used Quantum espresso version 6.3 for our calculations. Density functional theory estimated to be $3.25-3.27 \mathrm{eV}$ with respect to the $\mathrm{ZnO}$ film and the refractive index is.

(DFT) was used as as implemented in the PWSCF code ("Becke: Density-functional Thermochemistry. III.... - Google Scholar" n.d.). Exchange and correlation were treated with GGA using the Becke-Lee-Yang-Parr (BLYP) functional (Becke 1988). We used the ultra soft pseudopotentials: Zn.blypvan_ak.UPF, O.blyp-van_ak.UPF and Sr.blyp-sp-hgh.UPF("Pseudopotentials QUANTUMESPRESSO" n.d.). It is a well known fact that GGA approximation underestimates the band gap value in semiconductors ("Effect of Phase Transition on the Optoelectronic Properties of Zn1-xMgxS: Journal of Applied Physics: Vol 112, No 7" n.d.) ("Effect of Phase Transition on the Optoelectronic Properties of Zn1-xMgxS: Journal of Applied Physics: Vol 112, No 7" n.d.) (“Structural and Optoelectronic Properties of the Zinc Titanate Perovskite and Spinel by Modified Becke-Johnson Potential" 2013), but does not affect the accuracy of the comparison of the related properties of the crystals.

The supercell used as the starting point in the calculation was $2 \times 2 \times 1$ (16 atoms: $\mathrm{Zn}=8$ atoms and $\mathrm{O}=8$ atoms). Supercell is of the experimental unit cell of wurtzite $\mathrm{ZnO}$. The original lattice parameters are $a=b=3.2493$ $\AA$, c=5.2057 $\AA$ and space group P63mc (186) ("Journal of Applied Crystallography 10.1107/S1600576714003379-Google”

n.d.).

The first step in this simulation is applying self-consistent field (scf) calculation to the supercell (16 atoms) of $\mathrm{ZnO}$ to determine cutoff energy parameter and obtain the minimum energy. Figure 1 shows the resulting of calculation of cutoff plane-wave energy. The plane-wave expansion 
limit to point $40 \mathrm{Ry}$; accordingly the cutoff density for ultra soft psuedopotentials $(8 \times$ plane-wave cutoff energy) is set at $320 \mathrm{Ry}$. 8 zinc and 8 Oxygen atoms) was performed. The lattice parameter of the supercell is $\mathrm{a}=\mathrm{b}=6.4986 \AA, \mathrm{c}=5.2057 \AA$ and relaxed volume of the supercell was found to be $1361.6849 \mathrm{a}^{3}$; is the same as unrelaxed volume. A Sr-doped models ( $\mathrm{Sr}-\mathrm{ZnO})$ was developed from unit cell expansion of $\mathrm{ZnO}$ in the direction of axes $a, b$ as shown in figure 2, resulting at $12.5 \%, 25 \%, 37.5 \%$, $50 \%, 62.5 \%, 75 \%$ and $87.5 \%$ and $100 \%$ concentration. Table 1 shows the amount of percentage in term of atoms replacement. The band structure of the Wurtzite $\mathrm{ZnO}$ and its defect $\mathrm{Sr}-\mathrm{ZnO}$ was calculated along the various symmetry lines [G $(0.0,0.0,0.0), \mathrm{M}$ (0.0, 0.5, 0.0), L (0.0, 0.5, 0.5), A (0.0, 0.0, $0.5), \mathrm{H}(0.3,0.3,0.5), \mathrm{K}(0.3,0.3,0.0), \mathrm{M}$

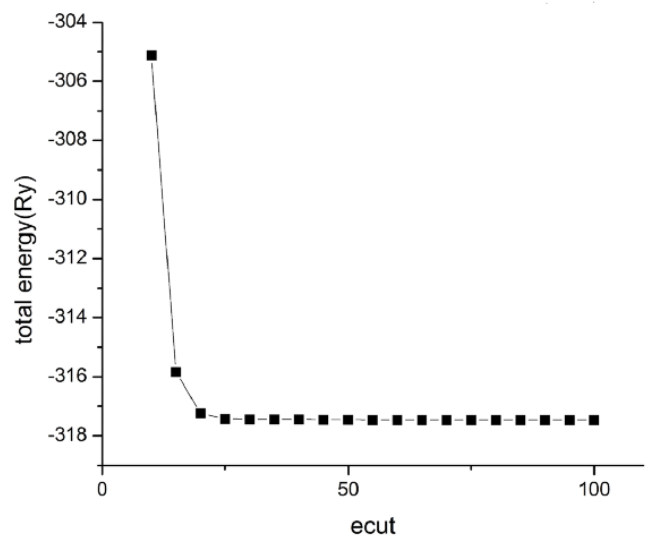

Figure 1: cutoff energy vs. total energy charge

Optimization of lattice parameters and generation of super cell contains 16 atoms ( $(0.0,0.5,0.0), \mathrm{G}(0.0,0.0,0.0)$, and $\mathrm{A}(0.0$, $0.0,0.5)]$.

\section{Results and Discussion}

\section{Band structure of pure $\mathrm{ZnO}$}

Band structure is an electronic structure in form of energy scheme to describe the conductivity of materials. The calculated band structure of pure $\mathrm{ZnO}$ crystal structure is presented along the various symmetry lines ( $\Gamma, \mathrm{M}, \mathrm{L}, \mathrm{A}, \mathrm{H}, \mathrm{K}, \mathrm{M}, \Gamma$, and $\mathrm{A})$ of the Brillouin zone in Figure 3. The Fermi level $E_{F}$ was set to zero; above and below this level were the conduction band $\mathrm{CB}$ and

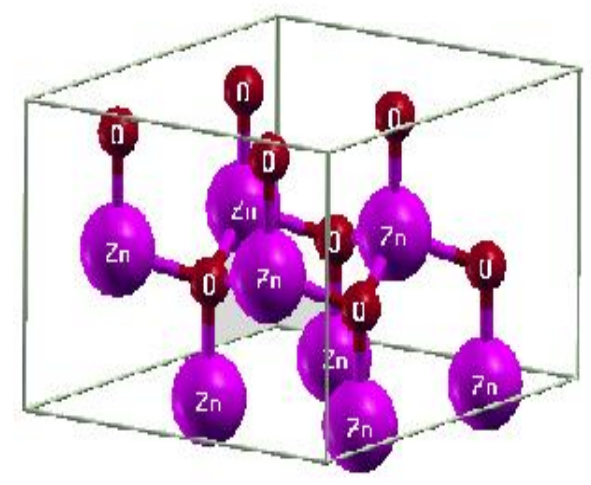

Figure2: 2x2x1 wurtzite supercell Crystalline structures for $\mathrm{Sr}: \mathrm{ZnO}$ materials. 
Table 1: The amount (in \%) of Sr doped on $\mathrm{ZnO}$ models used in simulation

\begin{tabular}{|c|c|}
\hline $\begin{array}{c}\text { No. of } \mathrm{Zn} \\
\text { atoms } \\
\text { replaced by } \mathrm{Sr}\end{array}$ & $\begin{array}{c}\text { Doped amount } \\
(\%)\end{array}$ \\
\hline 1 & 12.5 \\
\hline 2 & 25.0 \\
\hline 3 & 37.5 \\
\hline 4 & 50.0 \\
\hline 5 & 62.5 \\
\hline 6 & 75.0 \\
\hline 7 & 87.5 \\
\hline 8 & 100 \\
\hline
\end{tabular}

valence band VB, respectively. The minimum energy level of the conduction band CBM and the maximum energy level of the valence band VBM were at the same k-point $(\Gamma-\Gamma)$, indicating that the $\mathrm{ZnO}$ was a direct band gap semiconductor. The calculated band gap of the $\mathrm{ZnO}$ was 0.9349 $\mathrm{eV}$. This is a new result. To our knowledge nobody has been work in $\mathrm{ZnO}$ used the exchange correlation BLYP functional based on DFT.

\section{Indirect band gap}

Substitution of one zinc atom by strontium atom gives $12.5 \%$ concentration of $\mathrm{Sr}$ in $\mathrm{ZnO}$. The structure formula of this model is $\mathrm{Zn}_{7} \mathrm{Sr}_{1} \mathrm{O}_{8}$. The relaxed volume of the cell is 1455.0a.u $\mathrm{u}^{3}$ compare with 1361.6849 a.u $\mathrm{u}^{3}$ of pure $\mathrm{ZnO}$. This cell volume variation is caused by bond length increase after the doping process. The ionic radius of the dopant $\mathrm{Sr}^{2+}\left(\mathrm{RSr}^{2+}=1.18 \AA\right)$ is bigger than that of $\mathrm{Zn}^{2+}\left(\mathrm{R}_{\mathrm{Zn}}{ }^{2}+=0.74 \AA\right)$. When $\mathrm{Sr}$ occupies the $\mathrm{Zn}$ site, the repulsion between the $\mathrm{Zn}$ and $\mathrm{Sr}$ causes further expansion of the cell volume. In addition, the bond length of the $\mathrm{Sr}-\mathrm{O}$ is lengthier compared to that of
$\mathrm{Zn}-\mathrm{O}$, which results in the said expansion. Indirect band gap is predicted at $12.5 \%$, $25 \%, 37.5 \%$ and $50 \%$ concentration of $\mathrm{Sr}$ on $\mathrm{ZnO}$ material. Table2 shows different values of k-points and energy gap of those $\mathrm{Sr}-\mathrm{ZnO}$ models. Refer to table2, at $12.5 \%$ concentration, it can be notice that the conduction band minimum $(\mathrm{CBM})$ is at $\Gamma-$ point; While the valence band maximum (VBM) is at L-point. This indicates that the band gap in this model is indirect band gap semiconductor with band gap $\approx 1.38 \mathrm{eV}$. This is because the k-points are different. The picture is described in figure 4 . At $25 \%$ concentration we found that the conduction band minimum CBM) is at $\Gamma$ (Gamma) point while the valence band maximum (VBM) is at A-point indicating indirect band semiconductor with band gap $\approx 1.25 \mathrm{eV}$. The minimum (CBM) and maximum (VBM) k-points at $37.5 \%$ concentration for $\mathrm{Sr}-\mathrm{ZnO}$ material is at $\Gamma$ - point and A-point respectively with indirect band gap energy $\approx$ $1.79 \mathrm{eV}$. These different K-points of 37.5 concentrations were depicted at $50 \%$ concentration but with indirect band gap energy $\approx 2.32 \mathrm{eV}$. 


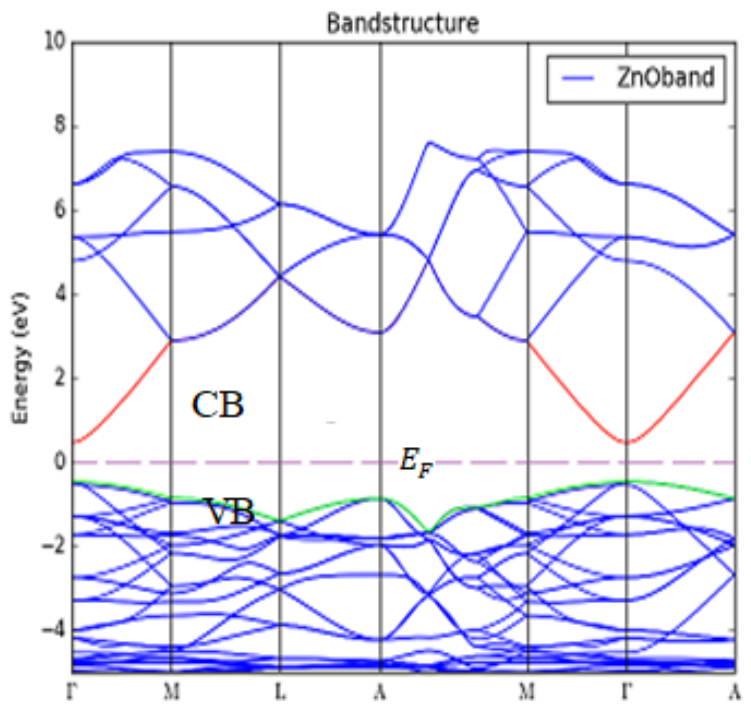

Figure3: Calculated pseudopotential band structure for pure wurtzite $\mathrm{ZnO}$ crystal. Red curve is CBM and the green one is VBM

Table2: conduction band mini, and valence band max., energy gap in $(\mathrm{eV})$ of different k-point for indirect band gap $\mathrm{Sr}-\mathrm{ZnO}$ models.

\begin{tabular}{|c|c|c|c|c|c|c|c|c|c|c|c|c|}
\hline \multirow[b]{3}{*}{$\begin{array}{c}\text { Doping } \\
\text { amount } \\
\text { (\%) }\end{array}$} & \multicolumn{12}{|c|}{ K-Points } \\
\hline & \multicolumn{3}{|c|}{$\Gamma-\Gamma$} & \multicolumn{3}{|c|}{ M-M } & \multicolumn{3}{|c|}{ L-L } & \multicolumn{3}{|c|}{ A-A } \\
\hline & $\begin{array}{c}\text { CBM } \\
(\mathrm{eV})\end{array}$ & $\begin{array}{l}\text { VBM } \\
(\mathrm{eV})\end{array}$ & $\begin{array}{c}\mathbf{E}_{\mathbf{g}} \\
(\mathbf{e V})\end{array}$ & $\begin{array}{c}\text { CBM } \\
(\mathrm{eV})\end{array}$ & $\begin{array}{c}\text { VBM } \\
(\mathrm{eV})\end{array}$ & $\begin{array}{c}\mathbf{E}_{\mathbf{g}} \\
(\mathbf{e V})\end{array}$ & $\begin{array}{c}\text { CBM } \\
(\mathrm{eV})\end{array}$ & $\begin{array}{l}\text { VBM } \\
(\mathrm{eV})\end{array}$ & $\begin{array}{c}\mathbf{E}_{\mathrm{g}} \\
(\mathrm{eV})\end{array}$ & $\begin{array}{c}\text { CBM } \\
(\mathrm{eV})\end{array}$ & $\begin{array}{l}\text { VBM } \\
(\mathrm{eV})\end{array}$ & $\begin{array}{c}\mathbf{E}_{\mathrm{g}} \\
(\mathrm{eV})\end{array}$ \\
\hline $12.5 \%$ & 0.6436 & -0.9036 & 1.55 & 2.6894 & -0.7639 & 3.45 & 4.0536 & -0.7340 & 4.79 & 2.7797 & -0.9707 & 3.75 \\
\hline $25 \%$ & 0.6934 & -0.8535 & 1.55 & 2.8203 & -1.1392 & 3.96 & 3.8842 & -0.8419 & 4.73 & 2.4503 & -0.5517 & 3.00 \\
\hline $37.5 \%$ & 0.8850 & -1.0844 & 1.97 & 2.6537 & -1.0825 & 3.74 & 3.7277 & -1.1645 & 4.90 & 2.6355 & -0.9048 & 3.54 \\
\hline $50 \%$ & 1.0940 & -1.2356 & 2.34 & 3.0663 & -1.3480 & 4.41 & 4.1933 & -1.4986 & 5.69 & 2.8823 & -1.2266 & 4.11 \\
\hline & & & & & & & & & & & & \\
\hline
\end{tabular}

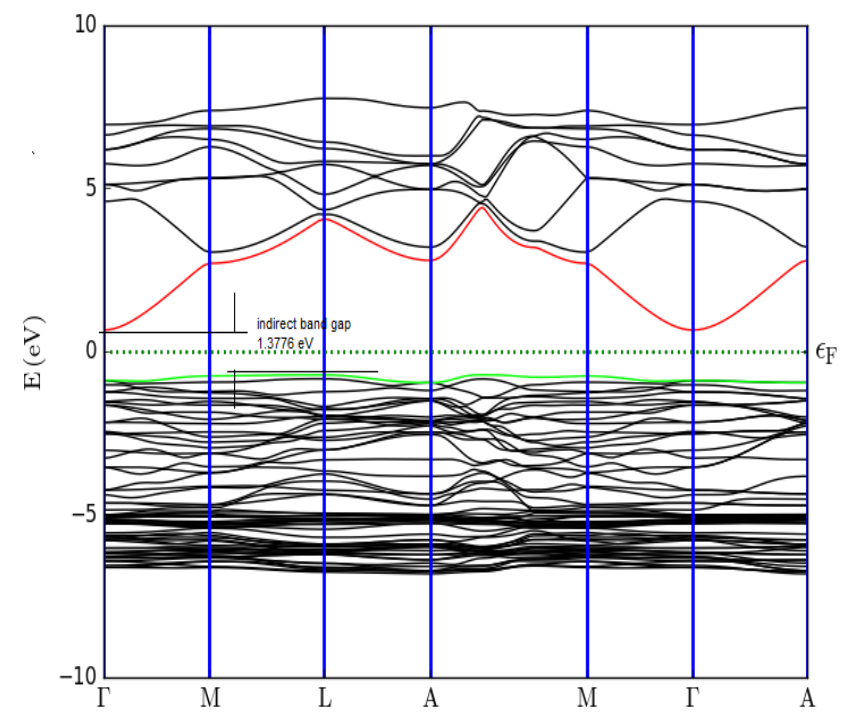


Figure4: Indirect band structure for $12.5 \% \mathrm{Sr}$ doped wurtzite $\mathrm{ZnO}$ crystal.

\section{Direct band gap structure}

The band structures of the $62.5 \%, 75 \%$, $87.5 \%$ and $100 \%$ concentrations of $\mathrm{Sr}$ in wurtizte $\mathrm{ZnO}$ have a direct band structure. Table3 shows k-point values those concentrations. The minimum conduction band (CBM) and maximum valence band $(\mathrm{VBM})$ are at the same k-points $\Gamma-\Gamma$ which revealed that the band structure for those concentration are a direct band structure. In direct band gap, the electron rising from valence band to conduction band will only change its potential, but in indirect band gap the electron rising from valence band to conduction band will change its potential and momentum.

Table3: conduction band mini, and valence band max., energy gap in (eV) of different k-point for direct band gap $\mathrm{Sr}-\mathrm{ZnO}$ models

\begin{tabular}{|c|c|c|c|c|c|c|c|c|c|c|c|c|}
\hline \multirow[b]{3}{*}{$\begin{array}{c}\text { Doping } \\
\text { amount } \\
(\%)\end{array}$} & \multicolumn{12}{|c|}{ K-Points } \\
\hline & \multicolumn{3}{|c|}{$\Gamma-\Gamma$} & \multicolumn{3}{|c|}{ M-M } & \multicolumn{3}{|c|}{ L-L } & \multicolumn{3}{|c|}{ A-A } \\
\hline & $\begin{array}{c}\text { CBM } \\
(\mathrm{eV})\end{array}$ & $\begin{array}{c}\text { VBM } \\
(\mathbf{e V})\end{array}$ & $\begin{array}{c}\mathbf{E}_{\mathbf{g}} \\
(\mathrm{eV})\end{array}$ & $\begin{array}{c}\text { CBM } \\
(\mathrm{eV})\end{array}$ & $\begin{array}{l}\text { VBM } \\
(\mathrm{eV})\end{array}$ & $\begin{array}{c}\mathbf{E}_{\mathbf{g}} \\
(\mathbf{e V})\end{array}$ & $\begin{array}{c}\text { CBM } \\
(\mathrm{eV})\end{array}$ & $\begin{array}{c}\text { VBM } \\
(\mathbf{e V})\end{array}$ & $\begin{array}{c}\mathbf{E}_{\mathbf{g}} \\
(\mathbf{e V})\end{array}$ & $\begin{array}{c}\text { CBM } \\
(\mathrm{eV})\end{array}$ & $\begin{array}{c}\text { VBM } \\
(\mathrm{eV})\end{array}$ & $\begin{array}{c}\mathbf{E}_{\mathbf{g}} \\
(\mathbf{e V})\end{array}$ \\
\hline $62.5 \%$ & 0.9327 & -0.7483 & 1.68 & 2.2241 & -0.8910 & 3.12 & 3.1812 & -1.5194 & 4.70 & 2.5547 & -1.4389 & 4.00 \\
\hline $75 \%$ & 1.4862 & -0.8942 & 2.38 & 3.2937 & -0.9577 & 4.25 & 4.1155 & -1.6271 & 5.74 & 3.0760 & -1.4364 & 4.51 \\
\hline $87.5 \%$ & 1.3150 & -1.0605 & 2.38 & 2.7507 & -1.2564 & 4.01 & 3.5498 & -2.1630 & 5.42 & 2.7610 & -1.8686 & 4.63 \\
\hline $100 \%$ & 2.2413 & -1.1272 & 3.37 & 2.8797 & 1.2268 & 4.11 & 4.2318 & -2.7048 & 6.94 & 4.2285 & -2.1091 & 6.34 \\
\hline
\end{tabular}

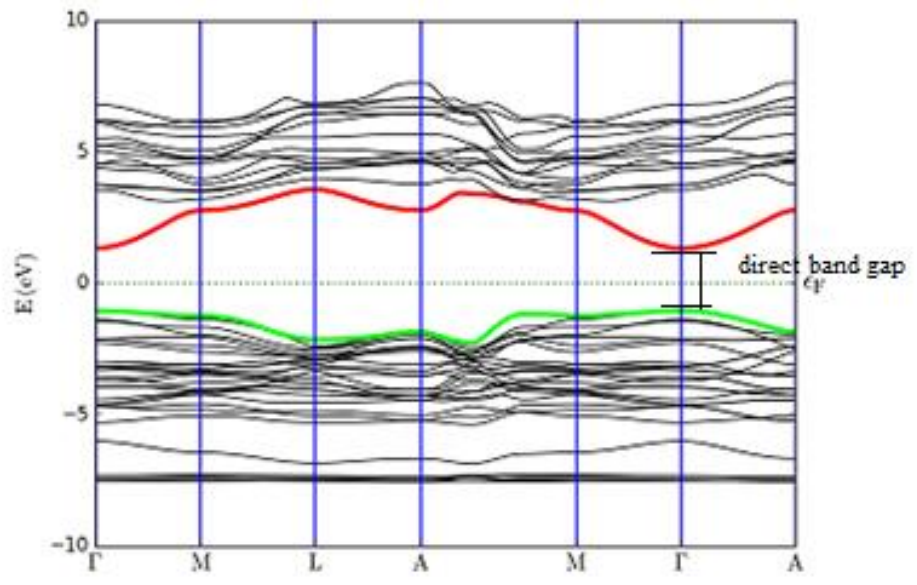

Figure4: direct band structure for $87.5 \% \mathrm{Sr}$ doped wurtzite $\mathrm{ZnO}$ crystal.

\section{Conclusion}

In conclusion, we have investigated the structural and electronic properties of pure and $\mathrm{Sr}$-doped $\mathrm{ZnO}$ using first principles density functional calculations. Band structure calculations were carried out using 
GGA-BLYP functional. The doping process presented increase in the band-gap energy $\mathrm{E}_{\mathrm{g}}$ at low percentages 25\%, 37.5\% and 50\% with indirect bang gap and direct band gap at high percentages $62.5 \%, 75 \%, 87.5 \%$ and $100 \%$.which we can called it wide indirect band gap. The change in the type of band structure by addition of strange atoms lead to the change in the properties of the original pure material which could be leads to new industrial applications.

\section{References}

Becke, Axel D. 1988. "Density-Functional Exchange-Energy Approximation with Correct Asymptotic Behavior." Physical Review A 38 (6): 3098.

"Becke: Density-functional Thermg ochemistry. III.... - Google Scholar." n.d. Accessed February 15, 2019.

"Effect of Phase Transition on the Optoelectronic Properties of Zn1-xMgxS: Journal of Applied Physics: Vol 112, No 7." n.d. Accessed August 31, 2019.

"Journal of Applied Crystallography بحث - 10.1107/S1600576714003379 Google." n.d. Accessed August 23, 2019.

Kalay, M., H. H. Kart, S. Özdemir Kart, and T. Çağın. 2009. "Elastic Properties and Pressure Induced Transitions of $\mathrm{ZnO}$ Polymorphs from FirstPrinciple Calculations." Journal of Alloys and Compounds 484 (1): 43138.

Klingshirn, Claus F., Andreas Waag, Axel Hoffmann, and Jean Geurts. 2010. Zinc Oxide: From Fundamental Properties towards Novel Applications. Vol. 120. Springer Science \& Business Media.

Mahmood, Asad, Fatih Tezcan, Gülfeza Kardaş, and Faruk Karadağ. 2017. "Effect of $\mathrm{Sr}$ Doping on the
Electronic Band Structure and Optical Properties of $\mathrm{ZnO}$ : A First Principle Calculation." Journal of Applied Physics 122 (11): 113102.

Mohamad, Ahmad Azmin, Muhammad Syafiq Hassan, Muhamad Kamil Yaakob, Mohamad Fariz Mohamad Taib, Fadhlul Wafi Badrudin, Oskar Hasdinor Hassan, and Muhd $\mathrm{Zu}$ Azhan Yahya. 2017. "FirstPrinciples Calculation on Electronic Properties of Zinc Oxide by ZincAir System." Journal of King Saud University - Engineering Sciences 29 (3): 278-83.

Moradian, Rostam, and Masoud Shahrokhi. 2013. "Structural, Electronic and Optical Properties of $\mathrm{Zn} 1-\mathrm{xSrxO}$ Nanotubes: First Principles Study." Journal of Physics and Chemistry of Solids 74 (8): 1063-68.

Mousavi, Seyed Javad. 2017. "FirstPrinciple Calculation of the Electronic and Optical Properties of Nanolayered ZnO Polymorphs by PBE and MBJ Density Functionals" 2 (4): 18.

"Pseudopotentials

QUANTUMESPRESSO.” n.d.

Accessed August 31, 2019.

https://www.quantum-

espresso.org/pseudopotentials.

Schreyer, M., L. Guo, S. Thirunahari, F. Gao, and M. Garland. 2014. "Simultaneous Determination of Several Crystal Structures from Powder Mixtures: The Combination of Powder X-Ray Diffraction, BandTarget Entropy Minimization and Rietveld Methods." Journal of Applied Crystallography 47 (2): 659-67.

Shen, L., R. Q. Wu, H. Pan, G. W. Peng, M. Yang, Z. D. Sha, and Y. P. Feng. 2008. "Mechanism of Ferromagnetism in Nitrogen-Doped 
ZnO: First-Principle Calculations." Physical Review B 78 (7): 073306.

"Structural and Optoelectronic Properties of the Zinc Titanate Perovskite and Spinel by Modified Becke-Johnson Potential." 2013. Physica B: Condensed Matter 420 (July): 5457.
Vijayan, T. A., R. Chandramohan, S. Valanarasu, J. Thirumalai, and S. P. Subramanian. 2008. "Comparative Investigation on Nanocrystal Structure, Optical, and Electrical Properties of $\mathrm{ZnO}$ and Sr-Doped $\mathrm{ZnO}$ Thin Films Using Chemical Bath Deposition Method." Journal of Materials Science 43 (6): 1776-82. 